\title{
Erratum to "Antisynchronization of a novel hyperchaotic system with parameter mismatch and external disturbances"
}

\author{
FEI YU ${ }^{1,2, *}, \mathrm{CHUNHUA} \mathrm{WANG}^{2}, \mathrm{YAN} \mathrm{HU}^{2}$ and JINWEN YIN ${ }^{2}$ \\ ${ }^{1}$ School of Computer and Communication Engineering, Changsha University of Science \\ and Technology, Changsha 410004, People's Republic of China \\ ${ }^{2}$ College of Information Science and Engineering, Hunan University, Changsha 410082, \\ People's Republic of China \\ *Corresponding author. E-mail: yufeiyfyf@qq.com
}

MS received 22 April 2013; revised 17 June 2013; accepted 29 August 2013

DOI: 10.1007/s12043-013-0624-2; $\boldsymbol{e}$ Publication: 27 November 2013

\begin{abstract}
In our recent publication (Pramana - J. Phys. 79, 81 (2012), DOI: 10.1007/s12043012-0285-6), our statement on adaptive antisynchronization (AS) controller design is incorrect. In this erratum we propose a correct control law. The corresponding proof is also given to demonstrate the effectiveness of the proposed control strategy.
\end{abstract}

Keywords. Hyperchaotic system; antisynchronization; parameter mismatch; external disturbances; adaptive control.

PACS Nos 05.45.Xt; 05.45.Pq; 05.45.Jn

\section{Introduction}

In our recent publication (Pramana - J. Phys. 79, 81 (2012)), our statement on adaptive antisynchronization (AS) controller (eq. (10)) is incorrect, because the control input had the unknown parameter vectors $\alpha$ and $\beta$ as also the disturbances $\mathbf{d}^{\prime}$ and $\mathbf{d}^{\prime \prime}$ were used. The vectors $\alpha$ and $\beta$ in eq. (10) should be replaced by $\hat{\alpha}$ and $\hat{\beta}$. Furthermore, we were wrong to use external disturbances in control input. The fact is that disturbances are unknown and very trivial and it is difficult to know disturbances exactly. Therefore, the proposed control input in the previous work is not applicable and realistic. In this erratum we propose a new control law which contains a compensator that is introduced to eliminate the influence of the external uncertainties. The corresponding proof is also given. 


\section{Design of a correct AS controller}

Consider the drive chaotic system and the response system in the form of

$$
\begin{aligned}
& \dot{x}=\mathbf{f}(x)+\mathbf{F}(x) \boldsymbol{\alpha}+\mathbf{d}^{\prime}, \\
& \dot{y}=\mathbf{g}(y)+\mathbf{G}(y) \boldsymbol{\beta}+\mathbf{U}+\mathbf{d}^{\prime \prime},
\end{aligned}
$$

where $x=\left(x_{1}, x_{2}, \ldots, x_{n}\right)^{T}$ and $y=\left(y_{1}, y_{2}, \ldots, y_{n}\right)^{T}$ are the state vectors, $\alpha=$ $\left(\alpha_{1}, \alpha_{2}, \ldots, \alpha_{m}\right)^{T}$ and $\beta=\left(\beta_{1}, \beta_{2}, \ldots, \beta_{p}\right)^{T}$ are the uncertain parameter vectors of the drive system and the response system respectively. $\mathbf{d}^{\prime}=\left(d_{1}^{\prime}, d_{2}^{\prime}, \ldots, d_{n}^{\prime}\right)^{T}$ and $\mathbf{d}^{\prime \prime}=$ $\left(d_{1}^{\prime \prime}, d_{2}^{\prime \prime}, \ldots, d_{n}^{\prime \prime}\right)^{T}$ are the exotic disturbances of systems (1) and (2) respectively, which satisfy the bounded conditions $\left\|d_{n}^{\prime}\right\| \leq \sigma_{n}$ and $\left\|d_{n}^{\prime \prime}\right\| \leq \rho_{n}$ for all $t$, where $\sigma=$ $\left(\sigma_{1}, \sigma_{2}, \ldots, \sigma_{n}\right)^{T}$ and $\boldsymbol{\rho}=\left(\rho_{1}, \rho_{2}, \ldots, \rho_{n}\right)^{T}$ are known positive constants. The control input vector $\mathbf{U}=\left(U_{1}, U_{2}, \ldots, U_{n}\right)^{T}$ is used to realize synchronization of systems (1) and (2). Let $\mathbf{e}=\left(e_{1}, e_{2}, \ldots, e_{n}\right)^{T}=x+y$ is the AS error vector.

Theorem 1. If the nonlinear controller $\boldsymbol{U}$ is taken as

$$
\begin{aligned}
\mathbf{U}= & -\mathbf{f}(x)-\mathbf{F}(x) \hat{\boldsymbol{\alpha}}+\operatorname{sgn}\left[\operatorname{diag}\left(e_{1}, e_{2}, \ldots, e_{n}\right)\right] \boldsymbol{\sigma}-\mathbf{g}(y)-\mathbf{G}(y) \hat{\boldsymbol{\beta}} \\
& +\operatorname{sgn}\left[\operatorname{diag}\left(e_{1}, e_{2}, \ldots, e_{n}\right)\right] \boldsymbol{\rho}-k \mathbf{e},
\end{aligned}
$$

where $\operatorname{sgn}(\cdot)$ denotes the sign function. $\sigma=\left(\sigma_{1}, \sigma_{2}, \ldots, \sigma_{p}\right)^{T}, \boldsymbol{\rho}=\left(\rho_{1}, \rho_{2}, \ldots, \rho_{\rho}\right)^{T}$, $\hat{\alpha}$ and $\hat{\beta}$ are estimations of the unknown parameters $\alpha$ and $\beta$, respectively and the adaptive laws of parameters are taken as

$$
\dot{\hat{\alpha}}=[\mathbf{F}(x)]^{T} \mathbf{e}, \quad \dot{\hat{\beta}}=[\mathbf{G}(y)]^{T} \mathbf{e} .
$$

Then the response system can antisynchronize the drive system globally and asymptotically.

Proof. From the above theorem, the error dynamical system can be obtained as

$$
\begin{aligned}
\dot{\mathbf{e}}= & \mathbf{F}(x)(\boldsymbol{\alpha}-\hat{\boldsymbol{\alpha}})+\mathbf{F}(y)(\boldsymbol{\beta}-\hat{\boldsymbol{\beta}})+\operatorname{sgn}\left[\operatorname{diag}\left(e_{1}, e_{2}, \ldots, e_{n}\right)\right] \boldsymbol{\sigma} \\
& +\operatorname{sgn}\left[\operatorname{diag}\left(e_{1}, e_{2}, \ldots, e_{n}\right)\right] \boldsymbol{\rho}-k \mathbf{e} .
\end{aligned}
$$

Let $\tilde{\alpha}=\alpha-\hat{\alpha}, \tilde{\beta}=\beta-\hat{\beta}$. The Lyapunov function candidate can be taken as

$$
\mathbf{V}=\frac{1}{2}\left(\mathbf{e}^{2}+\tilde{\alpha}^{2}+\tilde{\beta}^{2}\right)=\frac{1}{2}\left(\mathbf{e}^{T} \mathbf{e}+\tilde{\boldsymbol{\alpha}}^{T} \tilde{\boldsymbol{\alpha}}+\tilde{\boldsymbol{\beta}}^{T} \tilde{\boldsymbol{\beta}}\right) .
$$

The time derivative of $\mathbf{V}$ is along the trajectory of the error system and substituting eqs (3)-(5) into the following equation, yields

$$
\begin{aligned}
\dot{\mathbf{V}}= & \dot{\mathbf{e}}^{T} \mathbf{e}+\tilde{\boldsymbol{\alpha}}^{T} \dot{\tilde{\alpha}}+\tilde{\boldsymbol{\beta}}^{T} \dot{\tilde{\boldsymbol{\beta}}}=\dot{\mathbf{e}}^{T} \mathbf{e}-\tilde{\boldsymbol{\alpha}}^{T}[\mathbf{F}(x)]^{T} \mathbf{e}-\tilde{\boldsymbol{\beta}}^{T}[\mathbf{G}(y)]^{T} \mathbf{e} \\
= & -k \mathbf{e}^{T} \mathbf{e}-\mathbf{e}^{T} \mathbf{d}^{\prime}+\mathbf{e}^{T} \operatorname{sgn}\left[\operatorname{diag}\left(e_{1}, e_{2}, \ldots, e_{n}\right)\right] \boldsymbol{\sigma}-\mathbf{e}^{T} \mathbf{d}^{\prime \prime} \\
& +\mathbf{e}^{T} \operatorname{sgn}\left[\operatorname{diag}\left(e_{1}, e_{2}, \ldots, e_{n}\right)\right] \boldsymbol{\rho} .
\end{aligned}
$$


Let $\eta_{1}=\mathbf{e}^{T} \operatorname{sgn}\left[\operatorname{diag}\left(e_{1}, \ldots, e_{n}\right)\right] \sigma-\mathbf{e}^{T} \mathbf{d}^{\prime}, \eta_{2}=\mathbf{e}^{T} \mathbf{d}^{\prime \prime}-\mathbf{e}^{T} \operatorname{sgn}\left[\operatorname{diag}\left(e_{1}, \ldots, e_{n}\right)\right] \boldsymbol{\rho}$, where $\eta_{1}, \eta_{2} \in \mathbf{R}$ and $\eta_{2} \geq 0$. According to the definition and assumption of $\mathbf{d}^{\prime}, \mathbf{d}^{\prime \prime}$ and $\boldsymbol{\sigma}, \boldsymbol{\rho}$, it is guaranteed that $\eta_{1} \leq \eta_{2}$. Therefore,

$$
\dot{\mathbf{V}}=-k \mathbf{e}^{T} \mathbf{e}+\eta_{1}-\eta_{2} \leq-k \mathbf{e}^{T} \mathbf{e} \leq 0 .
$$

So $\dot{\mathbf{V}}$ is negative semi-definite, and since $\mathbf{V}$ is positive definite, it follows that $\mathbf{e} \in$ $L_{\infty}, \alpha, \beta \in L_{\infty}$. Thus, $\dot{\mathbf{e}} \in L_{\infty}$, and according to eq. (4), it can be obtained that

$$
\int_{0}^{t}\|\mathbf{e}\|^{2} \mathrm{~d} t=\int_{0}^{t} \mathbf{e}^{T} \mathbf{e} \mathrm{d} t \leq-\frac{1}{k} \int_{0}^{t} \dot{\mathbf{V}} \mathrm{d} t=\frac{1}{k}[\mathbf{V}(0)-\mathbf{V}(t)] \leq \frac{1}{k} \mathbf{V}(0) .
$$

Since $\mathbf{V}(0) \leq \infty$ and according to Barbalat's lemma, we have $\lim _{t \rightarrow \infty}\|\mathbf{e}(t)\|=0$, i.e. the error dynamical system (5) will be stabilized at the zero equilibrium asymptotically.

This completes the proof. 